\title{
Coherence-controlled holographic microscopy enabled recognition of necrosis as the mechanism of cancer cells death after exposure to cytopathic turbid emulsion
}

Jana Collakova Aneta Krizova Vera Kollarova Zbynek Dostal Michala Slaba Pavel Vesely Radim Chmelik 


\title{
Coherence-controlled holographic microscopy enabled recognition of necrosis as the mechanism of cancer cells death after exposure to cytopathic turbid emulsion
}

\author{
Jana Collakova, ${ }^{a, b, *}$ Aneta Krizova, ${ }^{a, b}$ Vera Kollarova, ${ }^{a}$ Zbynek Dostal, ${ }^{a, b}$ Michala Slaba, ${ }^{a, b}$ Pavel Vesely, ${ }^{a, \dagger}$ and \\ Radim Chmelik

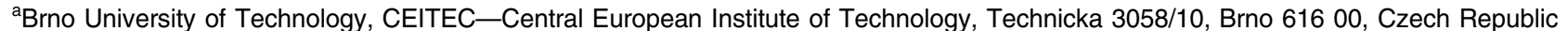 \\ ${ }^{\mathrm{b} B r n o}$ University of Technology, Faculty of Mechanical Engineering, Institute of Physical Engineering, Technicka 2896/2, Brno 616 00, \\ Czech Republic
}

\begin{abstract}
Coherence-controlled holographic microscopy (CCHM) in low-coherence mode possesses a pronounced coherence gate effect. This offers an option to investigate the details of cellular events leading to cell death caused by cytopathic turbid emulsions. CCHM capacity was first assessed in model situations that showed clear images obtained with low coherence of illumination but not with high coherence of illumination. Then, the form of death of human cancer cells induced by treatment with biologically active phospholipids (BAPs) preparation was investigated. The observed overall retraction of cell colony was apparently caused by the release of cell-to-substratum contacts. This was followed by the accumulation of granules decorating the nuclear membrane. Then, the occurrence of nuclear membrane indentations signaled the start of damage to the integrity of the cell nucleus. In the final stage, cells shrunk and disintegrated. This indicated that BAPs cause cell death by necrosis and not apoptosis. An intriguing option of checking the fate of cancer cells caused by the anticipated cooperative effect after adding another tested substance sodium dichloroacetate to turbid emulsion is discussed on grounds of pilot experiments. Such observations should reveal the impact and mechanism of action of the interacting drugs on cell behavior and fate that would otherwise remain hidden in turbid milieu. $\odot$ The Authors. Published by SPIE under a Creative Commons Attribution 3.0 Unported License. Distribution or reproduction of this work in whole or in part requires full attribution of the original publication, including its DOI. [DOI: 10.1117/1.JBO.20.11.111213]
\end{abstract}

Keywords: quantitative phase imaging; holographic microscopy; imaging through turbid media; coherence gate effect; live cell imaging, cancer cell necrotic death.

Paper 150165SSR received Mar. 15, 2015; published online Sep. 3, 2015.

\section{Introduction}

Generally, it is a difficult task for microscopic observation to study specimens in or covered with a turbid medium. This is particularly important if specimen changes are to be observed, such as activity and reactions of live cells. In this case, something more than phase contrast imaging is required. It has already been demonstrated that imaging through scattering media and turbid liquids is possible by digital holography. ${ }^{1-11}$ Separate holographic methods have been used for imaging through different turbid media, such as rotating diffuser, ${ }^{1}$ onion cell layer and cornea, ${ }^{2}$ milk, ${ }^{3,4}$ ground-glass diffuser, ${ }^{5-7}$ intralipid suspension, ${ }^{8}$ and biological tissue. ${ }^{9}$

However, a coherence-controlled holographic microscope 6 (CCHM) invented and built by our group provided low-coherence mode with improved coherence gate effect $^{7}$ (CGE). First, we examined this optical property in model situations. ${ }^{7,12}$ The positive experience obtained allowed the investigation of how preparation of biologically active phospholipids (BAPs) available only in turbid emulsion affects the cancer cells. BAPs

*Address all correspondence to: Jana Collakova, E-mail: jana.collakova@ ceitec.vutbr.cz

†Pavel Vesely and Radim Chmelik share senior authorship. are commonly considered to have positive influence on health. ${ }^{13}$ For the BAPs preparation, we used the anticancer activity that was already proved in the animal model in vivo and also in vitro. ${ }^{14}$ However, in the previous study, the mode of cancer cell death elicited by BAPs could not be established. This is an important aspect from the therapeutics point of view. Therefore, the aim of this study was to identify the course of events caused by the BAPs preparation that eventually leads to the determinantion of the cell death type.

\section{Material and Methods}

\subsection{Coherence-Controlled Holographic Microscope}

CCHM is an off-axis holographic system that interferometrically measures the optical phase shift of light passing through the specimen relative to the reference beam. ${ }^{6,15}$ The cell is a convenient thin and transparent specimen for this method. Nonaqueous components of the cell have similar specific refractive increments ${ }^{16}$ higher than water. Then, the amount of phase shift per pixel corresponds to cell dry mass distribution. ${ }^{16}$ From the quantitative information about phase shift in each pixel, the total dry mass of a whole cell can be calculated and expressed in picograms. ${ }^{17}$ Moreover, the changes of mass distribution with time bring invaluable information about cell motility. Consequently, 


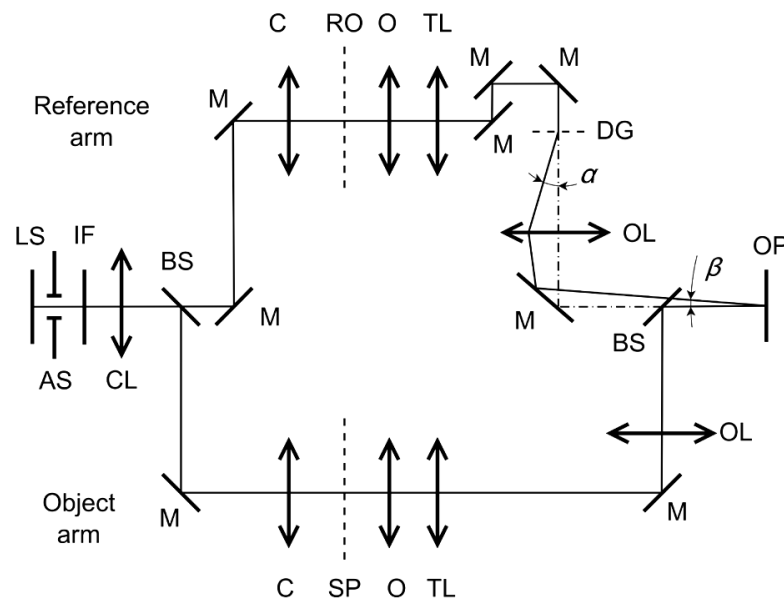

(a)

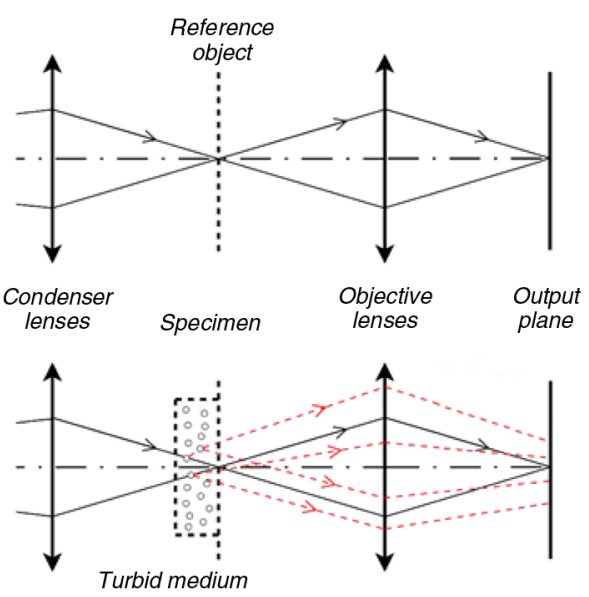

(b)

Fig. 1 (a) Optical setup of coherence-controlled holographic microscopy (CCHM). LS, light source; AS, aperture stop; IF, interference filter; CL, collector lens; BS, beam splitters; DG, diffraction grating; M, mirrors; C, condenser lenses; SP, specimen; RO, reference object; O, objectives; TL, tube lenses; OL, output lenses; OP, output plane. (b) Principle of imaging of the specimen with turbid medium. Nonballistic light (dashed lines) scattered in the output plane is not participating on the interference.

the leading noninvasive application of CCHM is the use of quantitative phase imaging (QPI) for measurement of dry mass changes during long-term evaluation of the living cells activity ${ }^{18,19}$ and exploitation of CGE for imaging in turbid milieu.

Optical setup of CCHM is shown in Fig. 1(a). The light from a light source (LS) passes through the aperture stop (AS) and interference filter (IF). These two elements enable selection of the degree of light coherence. Adjustable size of the aperture stop controls the spatial coherence, which substantially influences the imaging characteristics in transmission mode. Lateral resolution is better with a larger radius of the aperture stop, which corresponds to lower spatial coherence. Wider passband of the interference filter causes lower temporal coherence. These assumptions are fully described by Chmelik et al. ${ }^{12}$ and are generalized by Leith et al. ${ }^{20}$ As a result, imaging in transmitted-light setup is substantially affected by spatial coherence rather than temporal coherence (in contrast to optical coherence tomography ${ }^{2}$ ). Therefore, the interference filter, $\Lambda=650 \mathrm{~nm}$ (10 nm FWHM), was chosen as a nonphototoxic wavelength for live cell imaging.

After beam splitting, light passes through the achromatic interferometer [Fig. 1(a)] consisting of two separated optical paths - the object and the reference arm. Both arms are equipped by identical optical setups: condensers $(C)$, objectives $(O)$, and tube lenses (TL).

Off-axis holography is based on utilizing the first order of the diffraction grating (DG), ${ }^{12}$ which is placed in the reference arm. Object planes (SP, RO) and diffraction grating are optically conjugated with the output plane (OP). The interference fringe pattern, called a hologram in the output plane, is captured by CCD detector.

Critical specification of the optical elements requires precise selection of condenser and objective lenses to the setup to be optically identical. Following parameters of the system are crucial: identical optical path length in both arms and zero mutual shift of the object and reference beams in the output plane. These parameters are adjusted before every measurement, and they are maintained automatically to get the highest contrast of the interference fringes (i.e., imaging signal).
Observation through a scattering or turbid media [Fig. 1(b)] is possible due to CGE. ${ }^{6,7,12}$ Due to the possibility of using tunable degree of temporal and spatial coherence of light source, ${ }^{6}$ CCHM enables only the ballistic light (light that goes through specimen without being scattered) to interfere with the reference wavefront. The light that is scattered by specimen does not contribute to interference and, therefore, is filtered. Consequently, there is a limited contribution of light scattered in out-of-focus planes of the specimen to the image. As a result, an improved infocus image contrast arises and investigation of the intracellular dynamics is made possible.

\subsection{Cell Culture and Treatments}

Human colorectal adenocarcinoma cell line DLD-1 (ATCC ${ }^{\circledR}$ CCL221 ${ }^{\mathrm{TM}}$ ), kindly provided by AREKO Ltd., Prague, Czech Republic, and human breast adenocarcinoma cell line MCF-7 were used. Cells were cultivated at $37^{\circ} \mathrm{C}$ in a humidified incubator with $3.5 \% \mathrm{CO}_{2}$ in standard medium (Eagle's minimum essential medium based on Hanks balanced salt solution enriched with nonessential amino acids and $1 \mathrm{mM}$ sodium pyruvate, with $1 \mathrm{~g} \mathrm{NaHCO}_{3} / \mathrm{L}, 10 \%$ calf serum, $80 \mathrm{mM}$ gentamicin, and $2 \mathrm{mM}$ L-glutamine). Observation medium F10 was prepared without phenol red. $20 \mathrm{mM}$ TES (N-[tris(hydroxymethyl)methyl]-2-aminoethanesulfonic acid, 2-[(2-hydroxy-1, 1-bis(hydroxymethyl)ethyl)amino]ethanesulfonic acid) buffer (Sigma T5691) was used to set a pH of 7.4. For microscopy, trypsinized cells were seeded in subconfluent density and hermetically closed in the static observation chamber (Figs. 2, 3, 5, and 6), which consists of two coverslips and an annular ring spacer. The volume of the chamber is $0.7 \mathrm{ml}$ with $4 \mathrm{~mm}$ inner height for BAPs preparation in emulsion, which constitutes the strong diffusing layer between the coverslips. For observation in perfusion mode with online changes of medium (Fig. 4), the $\mu$-Slide I Leuer flow chamber (Ibidi Company) with $0.8 \mathrm{~mm}$ inner height was used. Cells were measured under standard laboratory conditions. A temperature of $37^{\circ} \mathrm{C}$ was constantly maintained in the environmental box for CCHM. 

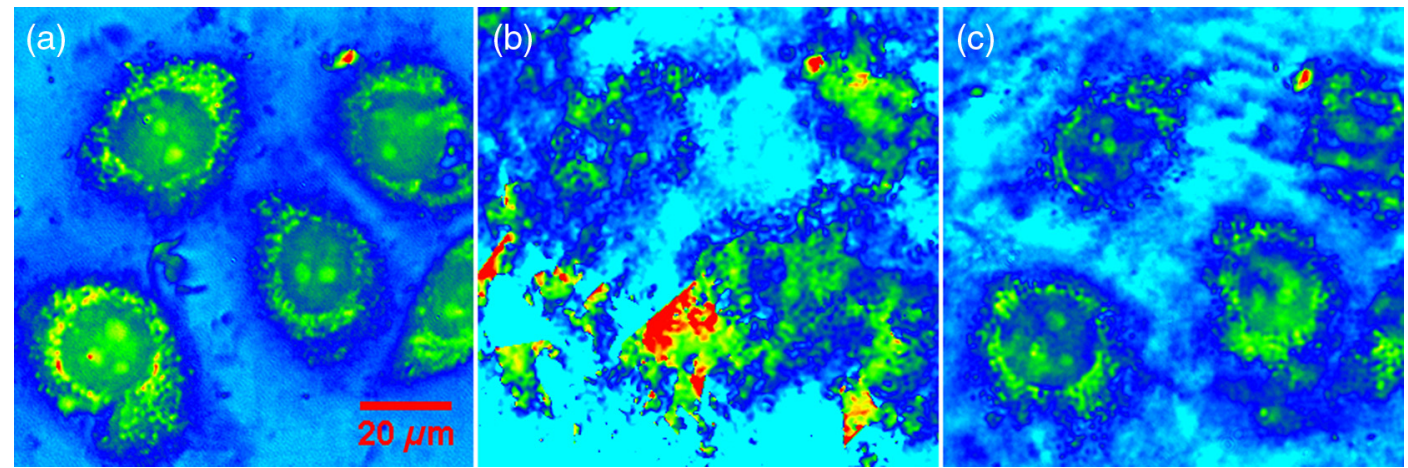

Fig. 2 Comparison of phase images of MCF-7 cells through turbid $0.1 \%$ biologically active phospholipids (BAPs) emulsion. CCHM equipped with (a) low-coherence illumination (halogen lamp), (b) coherent illumination without averaging (Fianium WhiteLase Supercontinuum laser), and (c) coherent illumination with average of 10 frames. The advantage of low-coherence illumination for a single-shot image of cells is clearly demonstrated. Pseudocolor quantitative phase imaging (QPI), objectives $20 \times / 0.5$.
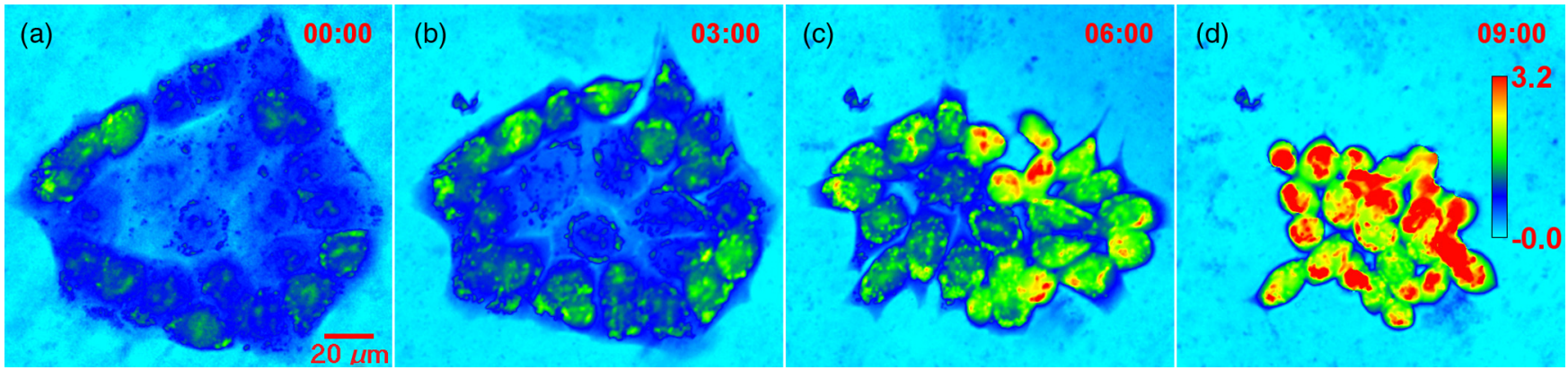

Fig. 3 Colony of human colorectal adenocarcinoma cells DLD-1 was followed in static chamber after exposure to $0.15 \%$ BAPs in culture medium. Three-hour intervals between (a) and (d) disclosed that apparent shrinkage of the whole colony began after $3 \mathrm{~h}$, followed by degradation of some cells not only at the edge of the colony but also inside, with clear condensation of all cell bodies in the colony marking their death. Pseudocolor QPI, calibration bar expresses dry mass density in pg $/ \mu \mathrm{m}^{2}$, objectives $20 \times / 0.5$.

BAPs were provided by AREKO Ltd., Prague, Czech Republic.

Sodium dichloroacetate (DCA, Sigma 347795) as a potential anticancer drug ${ }^{21}$ was dissolved in phosphate buffered saline ( $\mathrm{pH} 7.4)$, filtered, and diluted in cultivation medium.

\subsection{Criteria for Cell Death Evaluation}

Dynamic morphology of the treated cells that were observed led to the determination of cell death mode. ${ }^{22,23}$ Apoptosis, a programmed cell death, manifest features of dynamic morphology as a retraction of the cell into spherical shape that shows exaggerated zeiosis, which is a fast appearing and disappearing protrusions bulging at the tips and eventually splitting off the cell to form apoptotic bodies. Complete dismantling of the cell body is a desired trait of harmless removal of cell residues, because this does not initiate inflammation. Necrosis is an accidental cell death; dynamic morphology shows a variety of shape irregularities and damage to membrane integrity, such as blebs or blisters formation, which while bursting release cytoplasmic material, thus stimulating inflammatory response.

Assessment of the cell death mode was derived from the course of dynamic morphology events, not merely from appreciation of cell morphology registered by a single image.

\subsection{Image Acquisition and Processing}

Individual holograms are captured by camera Ximea MR4021MC-BH (maximum speed capture 5 fps by full resolution of $2048 \times 2018$ pixels). Captured holograms are processed in real time during the measuring process or in postprocessing procedure on PC (NVIDIA GeForce GTX 660, Intel Core i7, RAM 8 GB). The reconstruction of phase images from a captured hologram is based on carrier removal in the Fourier plane, ${ }^{24}$ and it is performed using self-developed software. During phase image processing, it is necessary to carry out the unwrapping procedure ${ }^{25}$ (Goldstein's branch-cut) and background compensation ${ }^{26}$ based on least-squares fitting. These algorithms compensate deformation in phase images and allow single-cell segmentation and cell tracking in time-lapse sequence. Reprocessing speed comes up to $25 \mathrm{fps}$ (GPU accelerated). All images in this paper represent resulting quantitative phase (QPI) captured by CCHM, and they have been adjusted to optimal contrast representation.

\section{Results and Discussion}

\subsection{Scattering Layer: Difference in Image Quality with Low and High Coherence of Illumination}

As mentioned earlier, reduction of spatial and temporal coherence allows for coherence gating. Spatially and temporally low- 
coherent illumination versus coherent illumination was used to obtain images of the same field of view. Figure 2 shows human breast adenocarcinoma cells MCF-7 embedded in 0.1\% BAPs emulsion (4 $\mathrm{mm}$ layer thickness). CCHM equipped with a halogen lamp provides noninvasive illumination of the sample not exceeding $0.2 \mu \mathrm{W} / \mathrm{cm}^{2}$ at the sample plane. In addition, the object beam was weakened through the turbid medium (scattering coefficient $\mu_{\mathrm{s}}=1 \mathrm{~mm}^{-1}$ ), and a neutral density filter was used in the reference arm to equilibrate intensity in both arms. The suppression of parasitic interferences is demonstrated in the case of low-coherent illumination even with single-shot acquisition [Fig. 2(a)] when a halogen lamp was used (interference filter $\Lambda=650 \mathrm{~nm}, 10 \mathrm{~nm}$ FWHM, coherence width $d_{\mathrm{w}}=$ $1.2 \mu \mathrm{m}$, and coherence length $d_{1}=42.2 \mu \mathrm{m}$ calculated in the object plane ${ }^{27}$ ). For demonstration of imaging with maximum coherence, it is necessary to use a laser because it is not possible to obtain point aperture stop in the setup. Therefore, the WhiteLase Supercontinuum laser with acousto-optic tunable filter $(\Lambda=650 \mathrm{~nm}, 4 \mathrm{~nm} \mathrm{FWHM})$ was used [Figs. 2(b) and 2(c)]. The phase image made by the laser without averaging [Fig. 2(b)] contains phase residues, ${ }^{24}$ which preclude the successful phase unwrapping procedure during image processing. The average of 10 images [Fig. 2(c)] was used to reduce speckle noise and multiple scattering on quasistatic turbid microfluidics of phospholipid particles. ${ }^{4}$ Spatial noise ${ }^{11}$ (fluctuations among the pixels in one image) was calculated as the standard deviation of pixels from the area that does not contain cells (background) in Fig. 1: panel (a) $\sigma=0.004 \mathrm{rad}(12 \mathrm{~nm})$, panel (b) $\sigma=$ $0.037 \mathrm{rad}(113 \mathrm{~nm})$, panel (c) $\sigma=0.013 \mathrm{rad}(40 \mathrm{~nm})$. This demonstrates that CCHM, especially if using low-coherent light, enables clear investigation of intracellular dynamics and reactions of cells to the treatment in high imaging quality and resolution (lateral resolution $900 \mathrm{~nm}$ by use of $20 \mathrm{x} / 0.5$ objectives).

\subsection{Cell Reactions to Biologically Active Phospholipids in Static Mode}

Colony of human colorectal adenocarcinoma cells DLD-1 (Fig. 3) offers two social situations for cells: First, it concerns cells along the colony edge, which are partly free from contacts with neighbor cells, and second, it deals with fully protected cells inside the colony. It was interesting to learn if there is a difference in the speed and efficiency of the BAPs-mediated damage. From Fig. 3, it is clear that cells around the edge were hit first, and the injury penetrated beyond them to steadily affect the whole colony. Considering this, it is not beyond reason that we in fact managed to mimic a similar course of events in an in vivo situation. Then, it may be worth considering to test screened anticancer drugs for their activity against in vitro colonies of growing and stationary cancer cells.

\subsection{Cell Reactions to Biologically Active Phospholipids in Perfusion Mode}

Cells of human breast adenocarcinoma line MCF-7 were planned to be repeatedly exposed to $0.5 \%$ BAPs in culture medium to see the course of events leading up to cell death (Fig. 4). In this way, necrosis or apoptosis can be determined. In Fig. 4, cells first show increasing of the fine granularity of cytoplasm and the coarse granularity around the nuclear membrane, then cessation of oscillating motion of perinuclear granules signals an irreversible step to cell death before the second application of BAPs [Fig. 4(e)]. Progressive deterioration of cells is speeded up by second BAPs challenge. This makes cells begin fast retraction due to rapidly releasing residual attachment to the culture substratum accompanied by an increasing amount of cell debris flowing in the medium. Loose cells, debris, and

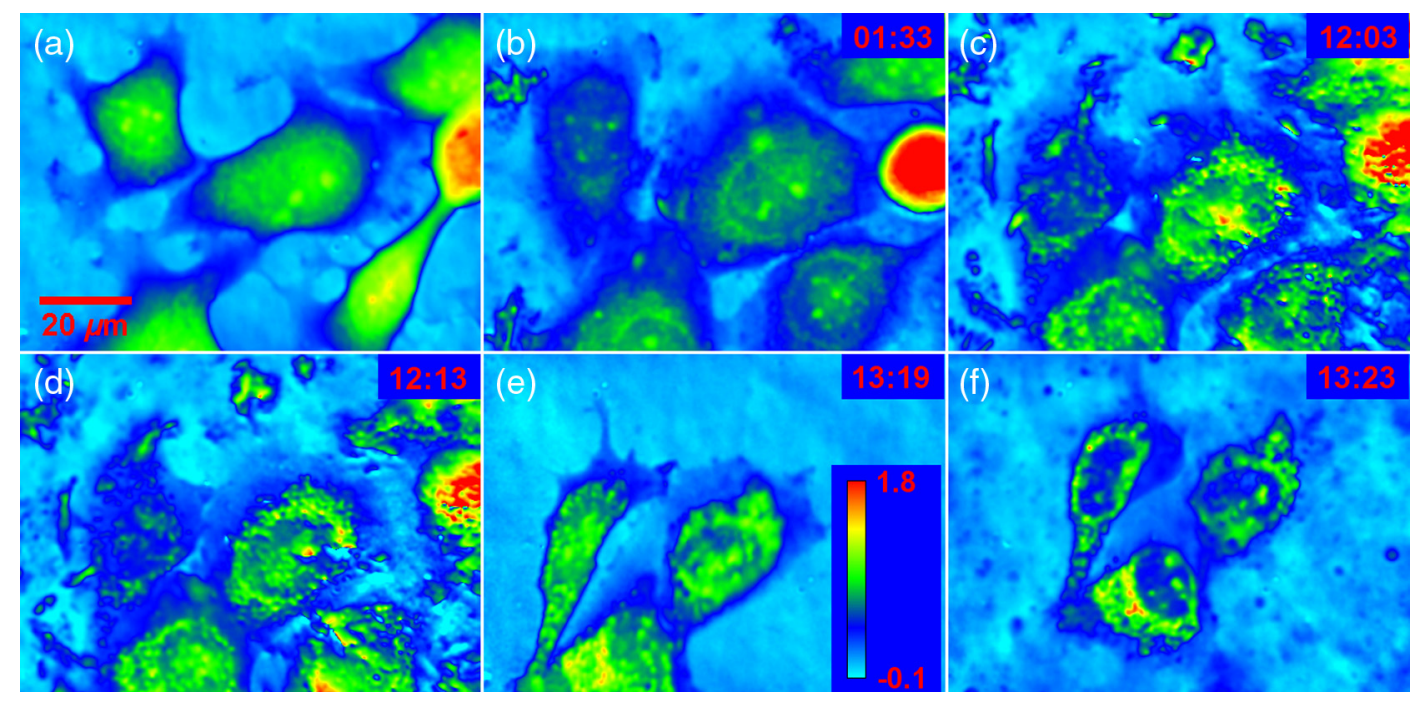

Fig. 4 Cells of human breast adenocarcinoma line MCF-7 were exposed to $0.5 \%$ BAPs in culture medium: (a) cells before the application of BAPs and (b) after first application (time label indicates period of the exposure in hours) overall increase of fine granularity and beginning of granular decoration of nuclear membrane on cytoplasmic side. (c) Granules around nucleus are ceasing their oscillating motion and (d) after $10 \mathrm{~min}$, cells started retraction due to rapidly releasing attachment to the culture substratum accompanied with increasing amount of cell debris. In (e), cells are exposed to next exchange of $0.5 \%$ BAPs in culture medium, which started washing out loose cells, debris, and dead cells from the remaining cells. In (f), final damage to cell membrane is manifested by vigorous formation of large and then bursting blisters, leading to cell death. Pseudocolor QPI, calibration bar expresses dry mass density in $\mathrm{pg} / \mu \mathrm{m}^{2}$, objectives $20 \times / 0.4$. 


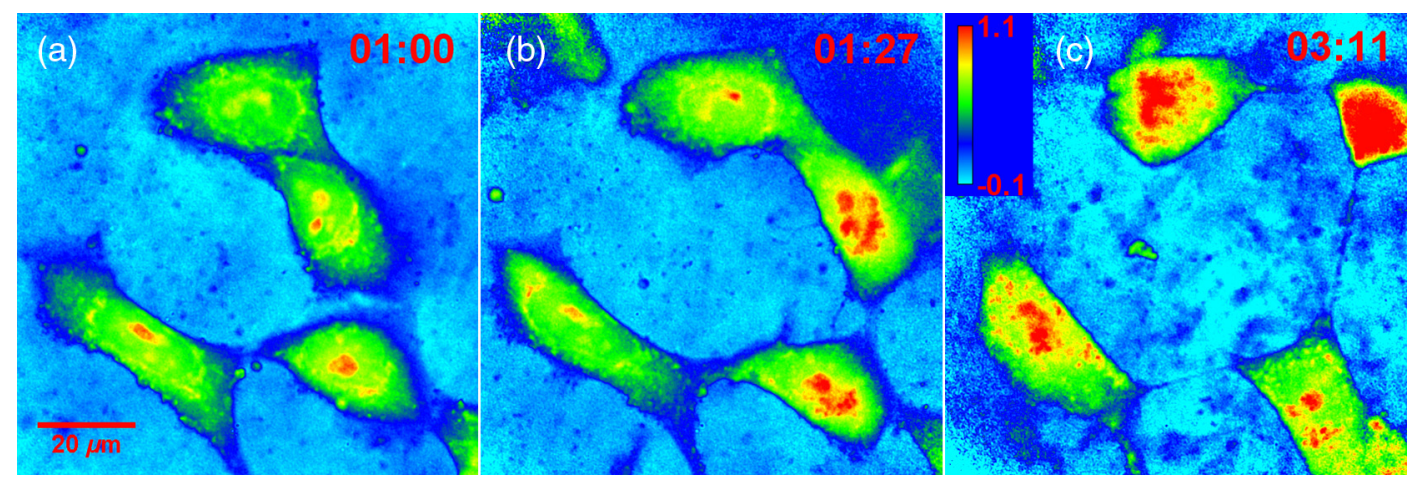

Fig. 5 Human colorectal adenocarcinoma cells DLD-1 treated with emulsion of $0.15 \%$ BAPs in culture medium: (a) cells in normal condition $1 \mathrm{~h}$ after the application of BAF, (b) decoration of nuclear membrane reveals beginning of nuclear membrane indentation, (c) followed by cell retraction and steady loss of cellular body integrity leading to cell death. Pseudocolor QPI, calibration bar expresses dry mass density in $\mathrm{pg} / \mu \mathrm{m}^{2}$, objectives $40 \times / 0.95$.

dead cells were washed out from the remaining cells. Final damage to the cell membrane is manifested by vigorous formation of large and bursting blisters leading to cell destruction by necrotic cell death.

\subsection{Details of Intracellular Reactions to Biologically Active Phospholipids}

Human colorectal adenocarcinoma cells DLD-1 treated with emulsion of $0.15 \%$ BAPs in culture medium and observed with objective lens 40×, NA 0.95 (Fig. 5), served a comparison with the effect of BAPs on MCF-7 cells. Again, decoration of nuclear membrane revealed the start of nuclear membrane indentation followed by cell body retractions, with decreasing cell integrity leading again to necrotic cell death.

\subsection{Pilot Experiment: Biologically Active Phospholipids in Combination with Dichloroacetate}

CCHM CGE offers an intriguing option of revealing the course of cell reactions to any drugs added to turbid media, which
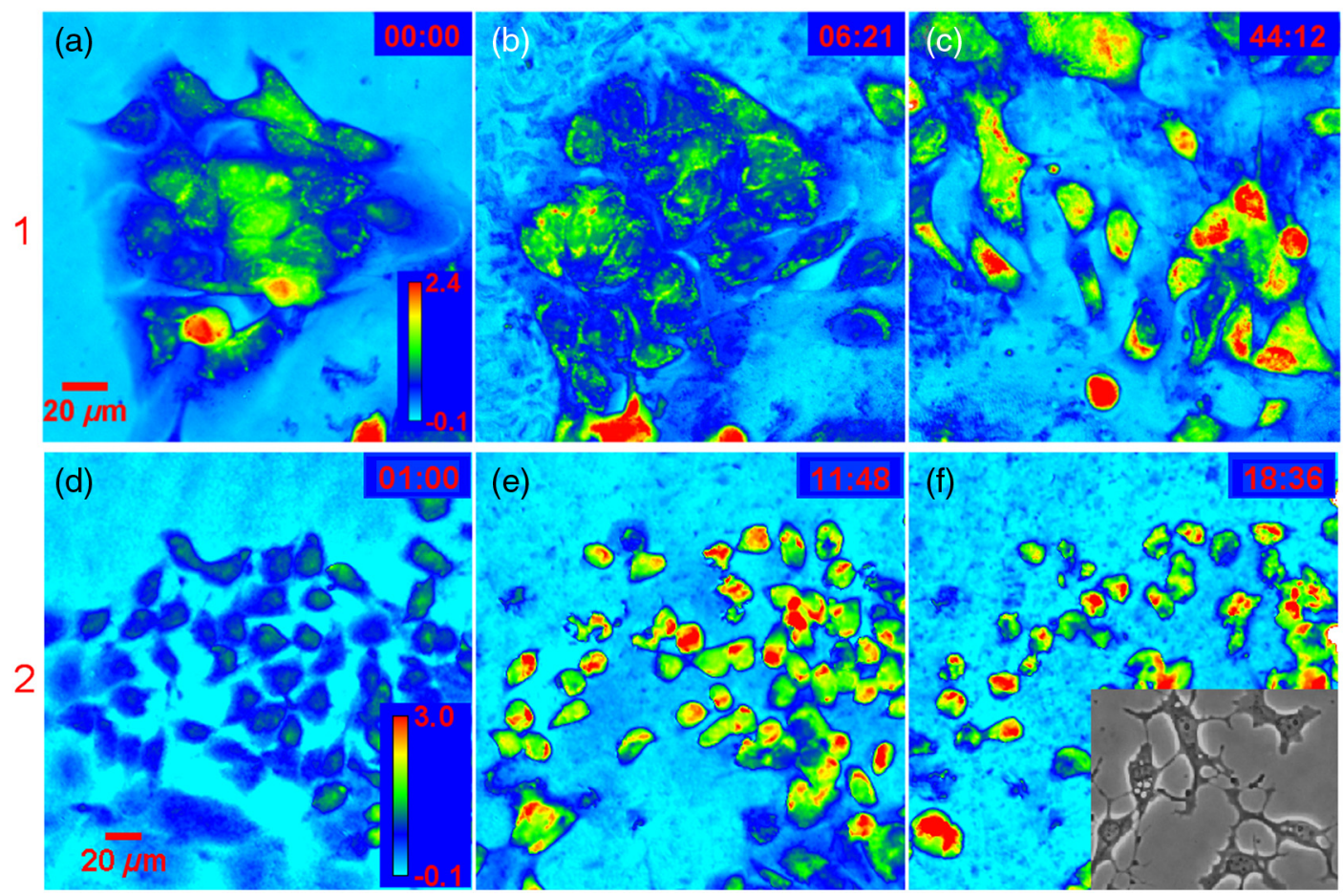

Fig. 6 First row shows human colorectal adenocarcinoma cells DLD-1 in $0.03 \%$ BAPs with added $20 \mathrm{mM}$ dichloroacetate (DCA): (a) starting conditions, (b) $6 \mathrm{~h}$ later granulation around the nucleus with slight deformation of nuclear membrane, (c) during the second progressive disintegration of cells without signs witnessing DCA contribution expressed as branching cell periphery, which is shown in insert in second row (in the lower right corner, treatment $80 \mathrm{mM}$ DCA). Second row shows MCF-7 in $0.15 \%$ BAPs: (d) after $1 \mathrm{~h}$ exposition, and damage of almost all cells after (e) $12 \mathrm{~h}$ and (f) $19 \mathrm{~h}$. There is no recognizable difference in damage to morphology between cells in the first and second rows. Pseudocolor QPI, calibration bar expresses dry mass density in $\mathrm{pg} / \mu \mathrm{m}^{2}$. 
would otherwise remain obscure because only the end result will be seen. The most interesting aspect of these drug combinations is uncovering a putative synergism against cancer cells. We are interested in learning if BAPs-caused necrosis could be modified to apoptosis, which is the desired cancer cell-induced death by therapeutic intervention. For this purpose, $0.03 \%$ BAPs emulsion was used with the addition of $20 \mathrm{mM}$ DCA to treat DLD-1 cells (Fig. 6). This is because DCA has been the focus of anticancer activity for some time $\mathrm{e}^{28}$ and is shown to induce apoptosis in colorectal adenocarcinoma cells. ${ }^{29}$ In spite of controverting reports, ${ }^{30}$ after recent results, ${ }^{31,32}$ DCA is being reconsidered for its anticancer activity. Regardless of technical feasibility, an expected contribution of DCA to the final damage to cells has not been observed yet. However, the methods of ongoing investigations will have to be further perfected.

\section{Conclusion}

CCHM, by providing CGE, makes single-shot registration of live cell activity in diffusive milieu possible. This advantage was used to determine the way BAPs have caused cancer cell death, whether programmed or accidental. In consequence, necrosis was identified as the mode of cell death elicited by BAPs preparation. We also attempted to examine possible synergism between BAPs and another drug added into the emulsion. In this case, it was DCA. The result of our preliminary experiment did not show any contribution of DCA to cell damage, at least at morphological level. However, using the technology for such examination was proven possible. This indicates a feasible practice for revealing cell activities that otherwise will remain unobserved.

\section{Acknowledgments}

This work was supported by the project Excellent Teams (CZ.1.07/2.3.00/30.0005) from the European Social Fund, by CEITEC - Central European Institute of Technology (CZ.1.05/ 1.1.00/02.0068) from the European Regional Development Fund, by Czech Science Foundation (15-14612S), and by a Specific Research grant (FSI/STI-J-15-2752) of Brno University of Technology.

\section{References}

1. M. Tziraki et al., "Photorefractive holography for imaging through turbid media using low coherence light," Appl. Phys. 70(1), 151-154 (2000).

2. P. Massatsch et al., "Time-domain optical coherence tomography with digital holographic microscopy," Appl. Opt. 44(10), 1806-1812 (2005).

3. S. Li and J. Zhong, "Dynamic imaging through turbid media based on digital holography," J. Opt Soc. Am 31(3), 480-486 (2014).

4. V. Bianco et al., "Clear coherent imaging in turbid microfluidics by multiple holographic acquisitions," Opt. Lett. 37(20), 4212-4214 (2012).

5. G. Indebetouw and P. Klysubun, "Imaging through scattering media with depth resolution by use of low-coherence gating in spatiotemporal digital holography," Opt. Lett. 25(4), 212-214 (2000).

6. T. Slaby et al., "Off-axis setup taking full advantage of incoherent illumination in coherence-controlled holographic microscope," Opt. Express 21(12), 14747-14762 (2013).

7. M. Lostak et al., "Coherence-controlled holographic microscopy in diffuse media," Opt. Express 22(4), 4180-4195 (2014).

8. Y. Pan, R. Birngruber, and R. Engelhardt, "Contrast limits of coherencegated imaging in scattering media," Appl. Opt. 36(13), 2979-2983 (1997).

9. E. Leith et al., "Imaging through scattering media with holography," J. Opt. Soc. Am. 9(7), 1148-1153 (1992).
10. M. Paturzo et al., "Microscopy imaging and quantitative phase contrast mapping in turbid microfluidic channels by digital holography," Lab Chip 12(17), 3073-3076 (2012).

11. G. Popescu, Quantitative Phase Imaging of Cells and Tissues, McGraw Hill, New York (2011).

12. R. Chmelik et al., "The role of coherence in image formation in holographic microscopy," Prog. Opt. 59(1), 267-336 (2014).

13. D. Kullenberg et al., "Health effects of dietary phospholipids," Lipids Health Dis. 11(3) (2012).

14. V. Karafiat, P. Vesely, and M. Dvorak, "Egg yolk phospholipids enriched with 1-O-Octadecyl-2-Oleoyl-sn-Glycero-3-Phospho-(NPalmitoyl) ethanolamine inhibit development of experimentally induced tumours," Folia Biol. 60(5), 220-227 (2014).

15. P. Kolman and R. Chmelik, "Coherence-controlled holographic microscope," Opt. Express 18(21), 21990-22003 (2010).

16. H. G. Davies et al., "The use of the interference microscope to determine dry mass in living cells and as a quantitative cytochemical method," J. Microsc. Sci. s3-95, 271-304 (1954).

17. A. F. Brown and G. A. Dunn, "Microinterferometry of the movement of dry matter in fibroblasts," J. Cell Sci. 92(3), 379-389 (1989).

18. H. Janeckova, P. Vesely, and R. Chmelik, "Proving tumour cells by acute nutritional/energy deprivation as a survival threat: a task for microscopy," Anticancer Res. 29(6), 2339-2345 (2009).

19. B. Rappaz et al., "Noninvasive characterization of the fission yeast cell cycle by monitoring dry mass with digital holographic microscopy," J. Biomed. Opt. 14(3), 034049 (2009).

20. E. N. Leith et al., "Optical sectioning by holographic coherence imaging: a generalized analysis," J. Opt. Soc. Am. A 20(2), 380-387 (2003).

21. S. Shahrzad et al., "Sodium dichloroacetate (DCA) reduces apoptosis in colorectal tumor hypoxia," Cancer Lett. 297(1), 75-83 (2010).

22. J. Balvan et al., "Multimodal holographic microscopy: distinction between apoptosis and oncosis," PLoS One 10(3), e0121674 (2015).

23. P. Golstein and G. Kroemer, "Cell death by necrosis: towards a molecular definition," Trends Biochem. Sci 32(1), 37-43 (2007).

24. T. Kreis, "Digital holographic interference-phase measurement using the Fourier-transform method," J. Opt. Soc. Am. A 3(6), 847-855 (1986).

25. D. C. Ghiglia and M. D. Pritt, Two-Dimensional Phase Unwrapping: Theory, Algorithms and Software, Wiley-Interscience, New York (1998).

26. T. Zikmund et al., "Sequential processing of quantitative phase images for the study of cell behaviour in real-time digital holographic microscopy," J. Microsc. 256(2), 117-125 (2014).

27. M. Born and E. Wolf, Principles of Optics, 7th ed., Cambridge University Press, Cambridge (1986).

28. E. D. Michelakis, L. Webster, and J. R. Mackey, "Dichloroacetate (DCA) as a potential metabolic-targeting therapy for cancer," $\mathrm{Br}$. $J$. Cancer 99(7), 989-994 (2008).

29. B. M. Madhok et al., "Dichloroacetate induces apoptosis and cell-cycle arrest in colorectal cancer cells," Br. J. Cancer 102(12), 1746-1752 (2010).

30. K. M. Anderson et al., "In vitro effects of dichloroacetate and $\mathrm{CO}_{2}$ on hypoxic HeLa cells," Anticancer Res. 29(11), 4579-4588 (2009).

31. Q. S. Chu et al., "A phase I open-labeled, single-arm, dose-escalation, study of dichloroacetate (DCA) in patients with advanced solid tumors," Invest New Drugs. 33(3), 603-610 (2014).

32. H. Shen et al., "Dual-targeting of aberrant glucose metabolism in glioblastoma," J. Exp. Clin. Cancer Res. 34(14) (2015).

Jana Collakova is a $\mathrm{PhD}$ student at the Brno University of Technology. She received her BS degree in optics from the Palacky University in Olomouc in 2009 and MS degree in optics and precise mechanics from the Brno University of Technology in 2011. Her current research interests include digital holographic microscopy in turbid media and living cells imaging.

Aneta Krizova is a PhD student at the Brno University of Technology. She received her BS and MS degrees in physical engineering from the Brno University of Technology in 2010 and 2012. Her current research interests include light microscopy, especially holographic microscopy and its applications.

Vera Kollarova is a postdoctoral fellow at the Brno University of Technology. She received her BS and MS degrees in optics from 
the Palacky University in Olomouc in 2001 and 2003, respectively, and $\mathrm{PhD}$ degree in optics from same university in 2011. Her current research interests include holographic microscopy and imaging through scattering media.

Zbynek Dostal is a PhD student at the Brno University of Technology. $\mathrm{He}$ received his $\mathrm{BS}$ degree in mechanical engineering in 2007 and MS degree in optics and precise mechanics in 2009 from the Brno University of Technology. $\mathrm{He}$ is a co-author of the CCHM patent for which he received the Werner von Siemens Excellence Award 2013. His current research interests include holographic microscopy, automation in microscopy, optical and mechanical design.

Michala Slaba is a PhD student at the Brno University of Technology. She received her $\mathrm{BS}$ and MS degrees in physical engineering from the Brno University of Technology in 2008 and 2010. Her current research interests include wave optics, imaging theory and holographic microscopy.
Pavel Vesely is a senior research scientist at the CEITEC, Brno University of Technology. He received his MD degree from the Charles University in Prague in 1961 and PhD degree in experimental biology from the Czechoslovak Academy of Sciences in 1965. He is the author of more than 100 journal papers. His lifelong research interests in cancer cell biology currently include domestication of coherence-controlled holographic microscopy in cell biology research.

Radim Chmelik is a professor of applied physics at the Brno University of Technology. He received his MS degree in solid state physics from the Masaryk University in Brno in 1989, and his PhD degree in physical and materials engineering from the Brno University of Technology in 1997. He is the author of more than 40 journal papers. His current research interests include wave optics, imaging theory, advanced and 3-D light microscopy, and holographic microscopy. He is a member of SPIE and OSA. 